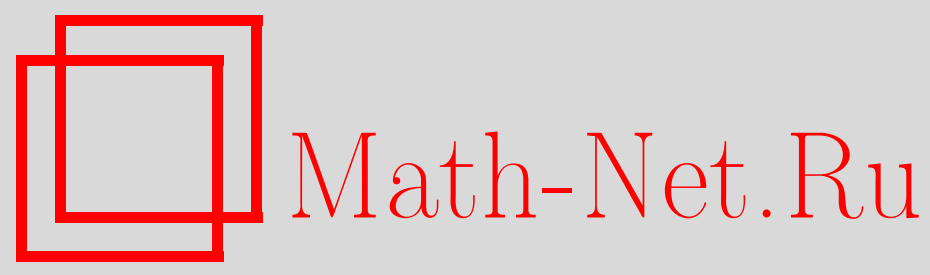

Г. В. Воскресенская, Метациклические группы и модулярные формы, Матем. заметки, 2000, том 67, выпуск 2, 163173

DOI: https://doi.org/10.4213/mzm825

Использование Общероссийского математического портала Math-Net.Ru подразумевает, что вы прочитали и согласны с пользовательским соглашением http://www. mathnet.ru/rus/agreement

Параметры загрузки:

IP : 54.166.219.16

26 апреля 2023 г., 02:29:35

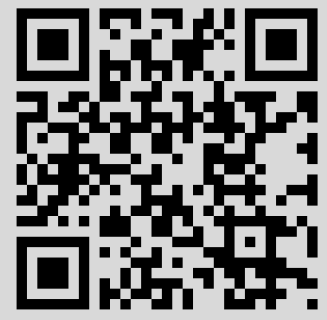


УДК 511.334

\section{МЕТАЦИКЛИЧЕСКИЕ ГРУППЫ И МОДУЛЯРНЫЕ ФОРМЫ}

\section{Г.В. Воскресенская}

В статье находятся все такие метациклические группы вида $\left\langle a, b: a^{m}=e, b^{s}=e\right.$, $\left.b^{-1} a b=a^{r}\right\rangle$, где $m=3,4,5,7,11,23$, что модулярные формы, ассоциированные со всеми элементами этих групп с помощью некоторого точного представления, являются мультипликативными $\eta$-произведениями.

Библиограффия: 4 названия.

1. Введение. Настоящая статья является продолжением исследований автора [1][4] проблемы нахождения таких конечных груп, что модулярные формы, ассоциированные со всеми элементами этих груп с помошью некоторого точного представления, принадлежат специальному классу модулярных форм, которыеназьваются мультипликативньпи $\eta$-произведениями. Исследуются метациклические групшы.

Напомним основные определения.

Эта-функиия Дедекинда $\eta(z)$ определяется формулой

$$
\eta(z)=q^{\frac{1}{24}} \prod_{n=1}^{\infty}\left(1-q^{n}\right), \quad q=e^{2 \pi i z}
$$

где $z$ лежит в верхней комплексной полуплоскости.

Мультипликативными $\eta$-произведениями назовем параболические формы целого веса с характерами, собственные относительно всех операторов Гекке и не имеющие нулей вне параболических вершин. Их ровно 28. Приведем их полньй список:

формы веса 1:

$$
\begin{gathered}
\eta(23 z) \eta(z), \quad \eta(22 z) \eta(2 z), \quad \eta(21 z) \eta(3 z), \quad \eta(20 z) \eta(4 z) \\
\eta(18 z) \eta(6 z), \quad \eta(16 z) \eta(8 z), \quad \eta^{2}(12 z)
\end{gathered}
$$

формы веса 2:

$$
\begin{aligned}
& \eta(15 z) \eta(5 z) \eta(3 z) \eta(z), \quad \eta(14 z) \eta(7 z) \eta(2 z) \eta(z), \quad \eta(12 z) \eta(6 z) \eta(4 z) \eta(2 z) \\
& \eta^{2}(11 z) \eta^{2}(z), \quad \eta^{2}(10 z) \eta^{2}(2 z), \quad \eta^{2}(9 z) \eta^{2}(3 z), \quad \eta^{2}(8 z) \eta^{2}(4 z), \quad \eta^{4}(6 z)
\end{aligned}
$$

формы веса 3:

$$
\eta^{2}(8 z) \eta(4 z) \eta(2 z) \eta^{2}(z), \quad \eta^{3}(7 z) \eta^{3}(z), \quad \eta^{3}(6 z) \eta^{3}(2 z), \quad \eta^{6}(4 z)
$$


формы веса 4:

$$
\eta^{4}(5 z) \eta^{4}(z), \quad \eta^{4}(4 z) \eta^{4}(2 z), \quad \eta^{2}(6 z) \eta^{2}(3 z) \eta^{2}(2 z) \eta^{2}(z), \quad \eta^{8}(3 z)
$$

форма веса 5: $\eta^{4}(4 z) \eta^{2}(2 z) \eta^{4}(z)$;

формы веса $6: \eta^{6}(3 z) \eta^{6}(z), \eta^{12}(2 z)$;

форма веса 8: $\eta^{8}(2 z) \eta^{8}(z)$;

форма веса $12: \eta^{24}(z)$.

К этому списку добавим еще две параболические формыполуцелого веса: $\eta(24 z), \eta^{3}(8 z)$.

Сопоставление элементам конечных групп модулярных форм осуществляется по следующему правилу. Пусть $\Phi$ - представление конечной группы $G$ унимодулярньми матрицами в пространстве $V$, размерность которого делится на 24 . Тогда для любого элемента $g \in G$ характеристический многочлен оператора $\Phi(g)$ имеет вид

$$
P_{g}(x)=\prod_{k=1}^{s}\left(x^{a_{k}}-1\right)^{t_{k}}, \quad a_{k} \in \mathbb{N}, \quad t_{k} \in \mathbb{Z} .
$$

С каждьм элементом $g \in G$ можно связать функцию

$$
\eta_{g}(z)=\prod_{k=1}^{s} \eta^{t_{k}}\left(a_{k} z\right)
$$

Функция $\eta_{g}(z)$ является параболической формой определенного уровня $N(g)$ и веса

$$
k(g)=\frac{1}{2} \sum_{k=1}^{s} t_{k}
$$

с характером, равным характеру квадратичного поля

$$
Q \sqrt{\prod_{k=1}^{s}\left(i a_{k}\right)^{t_{k}}}
$$

Метациклической группой назьвается конечная группа, имеющая циклический нормальный делитель, фактор-группа по которому также циклическая.

Генетический код метациклической группы: $\left\langle a, b: a^{m}=e, b^{s}=e, b^{-1} a b=a^{r}\right\rangle$.

Будем называть представление групшы искомым или представлением допустимого типа, если с помощью этого представления с элементами групшы ассоциируются мультипликативные $\eta$-произведения. Целью работы является нахождение метациклических групп, имеющих такие точные представления. При разборе каждого случая будут подробно указаны искомые представления.

Допустимые групшы указьваются с точностью до изоморфизма.

Искомые групш могут содержать элементы порядков, не превосходящих 24 и не равных 13, 17, 19. Непосредственно проверяется, что если некоторому элементу групш соответствует мультипликативное $\eta$-произведение, то всем его степеням также соответствуют параболические формы из указанного вьше списка. Используя этот факт, при 
исследовании груп достаточно рассматривать представления только для элементов, не лежащих в одной циклической группе. Единичному элементу групшы соответствует параболическая форма $\eta^{24}(z)$.

В следующей таблице приведены все возможности соответствия элементам циклических групп мультипликативных $\eta$-произведений.

\begin{tabular}{|c|c|}
\hline Групша & Параболические формы \\
\hline$Z_{24}$ & $\eta(24 z), \eta^{2}(12 z), \eta^{4}(6 z), \eta^{6}(4 z), \eta^{8}(3 z), \eta^{12}(2 z), \eta^{24}(z)$ \\
\hline$Z_{23}$ & $\eta(23 z) \eta(z), \eta^{24}(z)$ \\
\hline$Z_{22}$ & $\eta(22 z) \eta(2 z), \eta^{2}(11 z) \eta^{2}(z), \eta^{12}(2 z), \eta^{24}(z)$ \\
\hline$Z_{21}$ & $\eta(21 z) \eta(3 z), \eta^{3}(7 z) \eta^{3}(z), \eta^{8}(3 z), \eta^{24}(z)$ \\
\hline$Z_{20}$ & $\eta(20 z) \eta(4 z), \eta^{2}(10 z) \eta^{2}(2 z), \eta^{4}(5 z) \eta^{4}(z), \eta^{6}(4 z), \eta^{12}(2 z), \eta^{24}(z)$ \\
\hline$Z_{18}$ & $\eta(18 z) \eta(6 z), \eta^{2}(9 z) \eta^{2}(3 z), \eta^{3}(6 z) \eta^{3}(2 z), \eta^{12}(2 z), \eta^{24}(z)$ \\
\hline$Z_{16}$ & $\eta(16 z) \eta(8 z), \eta^{2}(8 z) \eta^{2}(4 z), \eta^{4}(4 z) \eta^{4}(2 z), \eta^{8}(2 z) \eta^{8}(z), \eta^{24}(z)$ \\
\hline$Z_{15}$ & $\eta(15 z) \eta(5 z) \eta(3 z) \eta(z), \eta^{4}(5 z) \eta^{4}(z), \eta^{6}(3 z) \eta^{6}(z), \eta^{24}(z)$ \\
\hline$Z_{14}$ & $\eta(14 z) \eta(7 z) \eta(2 z) \eta(z), \eta^{3}(7 z) \eta^{3}(z), \eta^{8}(2 z) \eta^{8}(z), \eta^{24}(z)$ \\
\hline$Z_{12}$ & $\begin{array}{c}\eta(12 z) \eta(6 z) \eta(4 z) \eta(2 z), \eta^{2}(6 z) \eta^{2}(3 z) \eta^{2}(2 z) \eta^{2}(z), \eta^{6}(3 z) \eta^{6}(z) \\
\eta^{8}(2 z) \eta^{8}(z), \eta^{24}(z), \eta^{4}(4 z) \eta^{2}(2 z) \eta^{4}(z)\end{array}$ \\
\hline$Z_{12}$ & $\eta^{2}(12 z), \eta^{4}(6 z), \eta^{6}(4 z), \eta^{8}(3 z), \eta^{12}(2 z), \eta^{24}(z)$ \\
\hline$Z_{11}$ & $\eta^{2}(11 z) \eta^{2}(z), \eta^{12}(2 z), \eta^{24}(z)$ \\
\hline$Z_{10}$ & $\eta^{2}(10 z) \eta^{2}(2 z), \eta^{4}(5 z) \eta^{4}(z), \eta^{12}(2 z), \eta^{24}(z)$ \\
\hline$Z_{9}$ & $\eta^{2}(9 z) \eta^{2}(3 z), \eta^{3}(6 z) \eta^{3}(2 z), \eta^{24}(z)$ \\
\hline$Z_{8}$ & $\eta^{2}(8 z) \eta(4 z) \eta(2 z) \eta^{2}(z), \eta^{4}(4 z) \eta^{2}(2 z) \eta^{4}(z), \eta^{8}(2 z) \eta^{8}(z), \eta^{24}(z)$ \\
\hline$Z_{8}$ & $\eta^{2}(8 z) \eta^{2}(4 z), \eta^{4}(4 z) \eta^{4}(2 z), \eta^{8}(2 z) \eta^{8}(z), \eta^{24}(z)$ \\
\hline$Z_{8}$ & $\eta^{3}(8 z), \eta^{6}(4 z), \eta^{12}(2 z), \eta^{24}(z)$ \\
\hline$Z_{7}$ & $\eta^{3}(7 z) \eta^{3}(z), \eta^{24}(z)$ \\
\hline$Z_{6}$ & $\eta^{2}(6 z) \eta^{2}(3 z) \eta^{2}(2 z) \eta^{2}(z), \eta^{6}(3 z) \eta^{6}(z), \eta^{8}(2 z) \eta^{8}(z), \eta^{24}(z)$ \\
\hline$Z_{6}$ & $\eta^{4}(6 z), \eta^{6}(4 z), \eta^{8}(3 z), \eta^{12}(2 z), \eta^{24}(z)$ \\
\hline$Z_{6}$ & $\eta^{3}(6 z) \eta^{3}(2 z), \eta^{6}(3 z) \eta^{6}(z), \eta^{8}(2 z) \eta^{8}(z), \eta^{24}(z)$ \\
\hline$Z_{5}$ & $\eta^{4}(5 z) \eta^{4}(z), \eta^{24}(z)$ \\
\hline$Z_{4}$ & $\eta^{4}(4 z) \eta^{2}(2 z) \eta^{4}(z), \eta^{8}(2 z) \eta^{8}(z), \eta^{24}(z)$ \\
\hline$Z_{4}$ & $\eta^{4}(4 z) \eta^{4}(2 z), \eta^{8}(2 z) \eta^{8}(z), \eta^{24}(z)$ \\
\hline$Z_{4}$ & $\eta^{6}(4 z), \eta^{12}(2 z), \eta^{24}(z)$ \\
\hline$Z_{3}$ & $\eta^{8}(3 z), \eta^{24}(z)$ \\
\hline$Z_{3}$ & $\eta^{3}(6 z) \eta^{3}(2 z), \eta^{24}(z)$ \\
\hline$Z_{2}$ & $\eta^{8}(2 z) \eta^{8}(z), \eta^{24}(z)$ \\
\hline$Z_{2}$ & $\eta^{12}(2 z), \eta^{24}(z)$ \\
\hline
\end{tabular}

Так как искомое представление является точньм, по виду параболической формы можно понять порядок соответствующего ей элемента группы. Поэтому мы будем приводить только списки параболических форм, указьвая элементы только в том случае, 
когда элементам группы равных порядков соответствуют различные формы.

Если некоторая допустимая группа является подгруппой другой допустимой метациклической группы, то можно привести подробное рассмотрение только для большей группы. Результат для подгрупшы следует из него очевидным образом.

В этой статье полностью разобраны случаи, когда $m=3,4,5,7,11,23$ и циклические подгрупшы $\langle a\rangle$ и $\langle b\rangle$ не имеют нетривиального пересечения.

Основной результат статьи может быть сформулирован в виде следующей теоремы.

ТЕОРемА. Пусть $G$ - такая метациклическая группа с генетическим кодом

$$
\left\langle a, b: a^{m}=e, b^{s}=e, b^{-1} a b=a^{r}\right\rangle, \quad m=3,4,5,7,11,23,
$$

что $с$ каждым әлементом әтой группы $с$ помощью некоторого точного представления ассочиируется мультипликативное $\eta$-произведение. Тогда для $m, s, r$ возмохны (с точностью до изоморфизма) лишь указанные в таблице значения:

\begin{tabular}{|c|c|c|c|c|c|c|c|c|c|c|c|c|c|c|c|c|c|c|c|}
\hline$m$ & 3 & 3 & 3 & 3 & 3 & 3 & 4 & 4 & 4 & 4 & 4 & 4 & 5 & 5 & 5 & 5 & 5 & 5 & 5 \\
\hline$s$ & 2 & 4 & 6 & 8 & 12 & 18 & 2 & 4 & 6 & 8 & 10 & 24 & 4 & 8 & 12 & 2 & 4 & 6 & 8 \\
\hline$r$ & 2 & 2 & 2 & 2 & 2 & 2 & 3 & 3 & 3 & 3 & 3 & 3 & 2 & 2 & 2 & 4 & 4 & 4 & 4 \\
\hline
\end{tabular}

\begin{tabular}{|c|c|c|c|c|c|c|c|c|c|c|c|c|c|c|c|c|}
\hline$m$ & 7 & 7 & 7 & 7 & 7 & 7 & 7 & 11 & 11 & 11 & 11 & 11 & 11 & 23 & 23 & 23 \\
\hline$s$ & 2 & 4 & 6 & 6 & 12 & 3 & 6 & 2 & 4 & 20 & 10 & 10 & 5 & 2 & 22 & 11 \\
\hline$r$ & 6 & 6 & 6 & 3 & 3 & 2 & 2 & 10 & 10 & 2 & 2 & 4 & 5 & 22 & 5 & 10 \\
\hline
\end{tabular}

Диэдральные группы допустимых типов подробно рассматривались автором в предыдущей статье [4].

2. Метациклические группы вида $\left\langle a, b: a^{3}=e, b^{s}=e, b^{-1} a b=a^{r}\right\rangle$. Имеем $m=3$.

В этом случае $r=2, s$ должно быть четным, $b^{s / 2}$ является центральным элементом. Допустимые порядки элементов искомой группы показывают, что в ней с элементами порядка 3 могут коммутировать элементы порядков $2,3,4,5,6,7,8,9,12,15,18,21,24$; $s / 2$ может равняться $2,3,4,5,6,7,8,9,12$.

2.1. Групша $\left\langle a, b: a^{3}=e, b^{4}=e, b^{-1} a b=a^{2}\right\rangle$. Это группа 12-го порядка; она имеет 6 классов сопряженных элементов.

Для этой групшы можно построить допустимые представления так, чтобы элементам порядка 6 соответствовали любые из трех параболических форм, соответствующие элементам порядка 6 .

Первым искомым представлением является прямая сумма

$$
2\left(T_{1} \oplus T_{2} \oplus T_{3} \oplus T_{4}\right) \oplus 4\left(T_{5} \oplus T_{6}\right),
$$

где

$$
\begin{array}{cl}
T_{k}(a)=1, & T_{k}(b)=i^{k}, k=1,4 \\
T_{5}(a)=T_{6}(a)=\left(\begin{array}{cc}
\zeta_{3} & 0 \\
0 & \zeta_{3}^{2}
\end{array}\right), & T_{5}(b)=\left(\begin{array}{cc}
0 & 1 \\
-1 & 0
\end{array}\right), \quad T_{6}(b)=\left(\begin{array}{ll}
0 & 1 \\
1 & 0
\end{array}\right) .
\end{array}
$$


Элементам групшы соответствуют параболические формы: $\eta^{4}(6 z), \eta^{8}(3 z), \eta^{6}(4 z)$, $\eta^{12}(2 z), \eta^{24}(z)$.

Вторьм искомым представлением является

$$
2\left(T_{1} \oplus T_{3} \oplus T_{5}\right) \oplus 4\left(T_{2} \oplus T_{3} \oplus T_{6}\right) .
$$

Элементам группы соответствуют параболические формы: $\eta^{2}(6 z) \eta^{2}(3 z) \eta^{2}(2 z) \eta^{2}(z)$, $\eta^{8}(3 z), \eta^{4}(4 z) \eta^{4}(2 z), \eta^{8}(2 z) \eta^{8}(z), \eta^{24}(z)$.

Третьим искомьм представлением является

$$
3\left(T_{1} \oplus T_{2} \oplus T_{3} \oplus T_{4} \oplus T_{5} \oplus T_{6}\right) .
$$

Элементам группы соответствуют параболические формы: $\eta^{3}(6 z) \eta^{3}(2 z), \eta^{8}(3 z)$, $\eta^{6}(4 z), \eta^{12}(2 z), \eta^{24}(z)$.

2.2. Групша $\left\langle a, b: a^{3}=e, b^{6}=e, b^{-1} a b=a^{2}\right\rangle$. Эта групша имеет 9 классов сопряженных элементов.

Искомым представлением является удвоенная прямая сумма всех неприводимых представлений.

Элементам $a, a^{2}$ соответствует форма $\eta^{6}(3 z) \eta^{6}(z)$, остальньм элементам третьего порядка соответствует форма $\eta^{8}(3 z)$, остальньм элементам группы соответствуют параболические формы $\eta^{4}(6 z), \eta^{12}(2 z), \eta^{24}(z)$.

2.3. Групша $\left\langle a, b: a^{3}=e, b^{8}=e, b^{-1} a b=a^{2}\right\rangle$. Это группа порядка 24 , она имеет 12 классов сопряженных элементов. Искомьм представлением является ее регулярное представление.

Элементам групшы соответствуют параболические формы: $\eta^{2}(12 z), \eta^{3}(8 z), \eta^{4}(6 z)$, $\eta^{8}(3 z), \eta^{6}(4 z), \eta^{12}(2 z), \eta^{24}(z)$.

2.4. Группа $\left\langle a, b: a^{3}=e, b^{12}=e, b^{-1} a b=a^{2}\right\rangle$. Эта группа порядка $36 ;$ у нее 18 классов сопряженных элементов.

Искомым представлением является сумма всех неприводимых представлений. Элементам, сопряженным с элементом $a b^{6}$, соответствует форма $\eta^{3}(6 z) \eta^{3}(2 z)$, элементам $a$ и $a^{2}$ соответствует форма $\eta^{6}(3 z) \eta^{6}(z)$. Остальным элементам групш соответствуют параболические формы $\eta^{2}(12 z), \eta^{4}(6 z), \eta^{6}(4 z), \eta^{8}(3 z), \eta^{12}(2 z), \eta^{24}(z)$.

2.5. Групша $\left\langle a, b: a^{3}=e, b^{18}=e, b^{-1} a b=a^{2}\right\rangle$. Искомое представление является суммой шести точных неизоморфных двумерньх представлений и удвоенной суммы шести одномерных представлений, при которых элемент $a$ переходит в единицу, а элемент $b$ - в различные степени первообразного корня степени 6 из 1.

Элементам группы соответствуют параболические формы: $\eta(18 z) \eta(6 z), \eta^{2}(9 z) \eta^{2}(3 z)$, $\eta^{3}(6 z) \eta^{3}(2 z), \eta^{6}(3 z) \eta^{6}(z), \eta^{8}(2 z) \eta^{8}(z), \eta^{24}(z)$.

3. Метациклические группы вида $\left\langle a, b: a^{4}=e, b^{s}=e, b^{-1} a b=a^{r}\right\rangle$. В этом случае $m=4$.

3.1. Групша $\left\langle a, b: a^{4}=e, b^{4}=e, b^{-1} a b=a^{3}\right\rangle$. Искомьм представлением является удвоенная прямая сумма всех неприводимых представлений.

Элементам $a, a^{3}, a b^{2}, a^{3} b^{2}$ соответствует $\eta^{4}(4 z) \eta^{4}(2 z)$, Элементу $a^{2}$ соответствует форма $\eta^{8}(2 z) \eta^{8}(z)$, остальным элементам 4 порядка соответствует $\eta^{6}(4 z)$, остальным элементам 2 порядка соответствует $\eta^{12}(2 z)$. 
3.2. Групша $\left\langle a, b: a^{4}=e, b^{6}=e, b^{-1} a b=a^{3}\right\rangle$. Эта группа является подгруппой в группе $\left\langle a, b: a^{4}=e, b^{24}=e, b^{-1} a b=a^{3}\right\rangle$.

3.3. Групша $\left\langle a, b: a^{4}=e, b^{10}=e, b^{-1} a b=a^{3}\right\rangle$. Искомое представление является прямой суммой всех неприводимых представлений, причем представления $T$, в которых собственными значениями оператора $T(b)$ являются только числа 1 и -1 , входят в сумму с кратностью 2, остальные представления с кратностью 1. Искомое представление находится однозначно.

Элементам группы соответствуют параболические формы: $\eta^{2}(10 z) \eta^{2}(2 z), \eta^{4}(5 z) \times$ $\eta^{4}(z), \eta^{6}(4 z), \eta^{12}(2 z), \eta^{24}(z)$.

3.4. Группа $\left\langle a, b: a^{4}=e, b^{24}=e, b^{-1} a b=a^{3}\right\rangle$. Эта группа порядка 96, в ней 48 классов сопряженных элементов.

Искомое представление является прямой суммой следующих 18 неприводимых представлений:

$$
\begin{aligned}
& T_{k}(a)=(-1)^{k}, \quad T_{k}(b)=\zeta_{12}^{k}, \quad 1 \leqslant k \leqslant 12 \\
& \Phi_{l}(a)=\left(\begin{array}{cc}
i & 0 \\
0 & -i
\end{array}\right), \quad \Phi_{l}(b)=\left(\begin{array}{cc}
0 & \zeta_{24}^{l} \\
\zeta_{24}^{l} & 0
\end{array}\right),
\end{aligned}
$$

$l=1,3,5,7,9,11$.

Элементам грушшы соответствуют параболические формы: $\eta(24 z), \eta^{2}(12 z), \eta^{4}(6 z)$, $\eta^{6}(4 z), \eta^{8}(3 z), \eta^{12}(2 z), \eta^{24}(z)$. Эта групша содержит в качестве подгруппы групу: $\left\langle a, b: a^{4}=e, b^{8}=e, b^{-1} a b=a^{3}\right\rangle$.

4. Метациклические группы вида $\left\langle a, b: a^{5}=e, b^{s}=e, b^{-1} a b=a^{r}\right\rangle$. Имеем $m=5, r=2$.

4.1. Метациклическая групша $\left\langle a, b: a^{5}=e, b^{4}=e, b^{-1} a b=a^{2}\right\rangle$. Эта групша порядка 20, у нее 5 классов сопряженных элементов. Вьпишем все ее 5 неприводимых представлений:

$$
\begin{aligned}
& T_{k}(a)=1, \quad T_{k}(b)=i^{k}, \quad k=1, \ldots, 4, \\
& T_{5}(a)=\left(\begin{array}{cccc}
\zeta_{5} & 0 & 0 & 0 \\
0 & \zeta_{5}{ }^{2} & 0 & 0 \\
0 & 0 & \zeta_{5}{ }^{3} & 0 \\
0 & 0 & 0 & \zeta_{5}{ }^{4}
\end{array}\right), \quad T_{5}(b)=\left(\begin{array}{cccc}
0 & 0 & 0 & 1 \\
1 & 0 & 0 & 0 \\
0 & 1 & 0 & 0 \\
0 & 0 & 1 & 0
\end{array}\right) \text {. }
\end{aligned}
$$

Первьм искомым представлением является прямая сумма

$$
2\left(T_{1} \oplus T_{2} \oplus T_{3} \oplus T_{4}\right) \oplus 4 T_{5} .
$$

Вторым искомым представлением является прямая сумма

$$
2 T_{1} \oplus 6 T_{4} \oplus 4 T_{5} .
$$

Третьим искомьм представлением является прямая сумма

$$
4\left(T_{2} \oplus T_{4} \oplus T_{5}\right) .
$$

Во всех трех случаях элементам пятого порядка соответствует $\eta^{4}(5 z) \eta^{4}(z)$, элементам четвертого порядка соответствует в первом случае $\eta^{6}(4 z)$, во втором случае $\eta^{4}(4 z) \times$ $\eta^{2}(2 z) \eta^{4}(z)$, в третьем случае $\eta^{4}(4 z) \eta^{4}(2 z)$; элементам второго порядка соответствует в первом случае $\eta^{12}(2 z)$, во втором и третьем случаях - форма $\eta^{8}(2 z) \eta^{8}(z)$. 
4.2. Метациклическая грушпа $\left\langle a, b: a^{5}=e, b^{8}=e, b^{-1} a b=a^{2}\right\rangle$. Эта группа порядка 40 , у нее 10 классов сопряженных элементов.

Искомым представлением является прямая сумма, в которую все двумерные неприводимые представления входят с кратностью 2, а одномерные неприводимые представления входят с кратностью 1.

Элементам группы соответствуют параболические формы: $\eta(20 z) \eta(4 z), \eta^{2}(10 z) \eta^{2}(z)$, $\eta^{6}(4 z), \eta^{4}(5 z) \eta^{4}(z), \eta^{8}(3 z), \eta^{12}(2 z), \eta^{24}(z)$.

Эта группа содержит в качестве подгруппы метациклическую группу $\left\langle a, b: a^{5}=e\right.$, $\left.b^{4}=e, b^{-1} a b=a^{4}\right\rangle$.

4.3. Метациклическая групша $\left\langle a, b: a^{5}=e, b^{12}=e, b^{-1} a b=a^{2}\right\rangle$. Эта группа порядка 60 , у нее 15 классов сопряженных элементов.

Единственным искомым представлением является представление

$$
T_{1} \oplus T_{2} \oplus T_{4} \oplus T_{5} \oplus T_{8} \oplus T_{9} \oplus 2\left(T_{3} \oplus T_{6} \oplus T_{7}\right),
$$

где

$$
\begin{aligned}
& T_{k}(a)=1, \quad T_{k}(b)=\zeta_{6}{ }^{k}, \quad k=1, \ldots, 6, \\
T_{7}(a)= & \left(\begin{array}{cccc}
\zeta_{5} & 0 & 0 & 0 \\
0 & \zeta_{5}^{2} & 0 & 0 \\
0 & 0 & \zeta_{5}{ }^{3} & 0 \\
0 & 0 & 0 & \zeta_{5}{ }^{4}
\end{array}\right), \quad T_{7}(b)=\left(\begin{array}{cccc}
0 & 0 & 0 & 1 \\
1 & 0 & 0 & 0 \\
0 & 1 & 0 & 0 \\
0 & 0 & 1 & 0
\end{array}\right), \\
& T_{8}=T_{2} \otimes T_{7}, \quad T_{9}=T_{4} \otimes T_{7} .
\end{aligned}
$$

Элементам групшы соответствуют параболические формы: $\eta(15 z) \eta(5 z) \eta(3 z) \eta(z)$, $\eta(12 z) \eta(6 z) \eta(4 z) \eta(2 z), \quad \eta^{2}(6 z) \eta^{2}(3 z) \eta^{2}(2 z) \eta^{2}(z), \quad \eta^{4}(4 z) \eta^{2}(2 z) \eta^{4}(z), \quad \eta^{6}(3 z) \eta^{6}(z)$ $\eta^{8}(2 z) \eta^{8}(z)$.

Эта групша содержит в качестве подгруппы метациклическую группу $\left\langle a, b: a^{5}=e\right.$, $\left.b^{6}=e, b^{-1} a b=a^{4}\right\rangle$.

4.4. Метациклическая группа $\left\langle a, b: a^{5}=e, b^{8}=e, b^{-1} a b=a^{4}\right\rangle$. Эта группа порядка 40, у нее 16 классов сопряженных элементов.

Искомым представлением является прямая сумма, в которую все двумерные неприводимые представления входят с кратностью 2, а одномерные неприводимые представления входят с кратностью 1.

Элементам группы соответствуют параболические формы: $\eta(20 z) \eta(4 z), \eta^{2}(10 z) \eta^{2}(z)$, $\eta^{6}(4 z), \eta^{4}(5 z) \eta^{4}(z), \eta^{8}(3 z), \eta^{12}(2 z), \eta^{24}(z)$.

5. Метациклические группы вида $\left\langle a, b: a^{7}=e, b^{s}=e, b^{-1} a b=a^{r}\right\rangle$.

5.1. Метациклическая групша вида $\left\langle a, b: a^{7}=e, b^{4}=e, b^{-1} a b=a^{6}\right\rangle$. Эта группа порядка 28, она имеет 10 классов сопряженных элементов.

Первым искомьм представлением является прямая сумма, в которую представления, в которых образ элемента $b$ имеет порядок 4 , входят с кратностью 1 , остальные неприводимые представления входят с кратностью 2.

Элементам групшы соответствуют параболические формы: $\eta(14 z) \eta(7 z) \eta(2 z) \eta(z)$, $\eta^{3}(7 z) \eta^{3}(z), \eta^{4}(4 z) \eta^{4}(2 z), \eta^{8}(2 z) \eta^{8}(z), \eta^{24}(z)$. 
Вторьм искомым представлением является прямая сумма, в которую входят все неприводимые представления, кроме тех одномерных, которые переводят элемент $b$ в -1 , причем точные двумерные неприводимые представления входят с кратностью 1 , неточные двумерные неприводимые представления входят с кратностью 2, единичное представление входит с кратностью 4. Элементам групшы соответствуют параболические формы: $\eta(14 z) \eta(7 z) \eta(2 z) \eta(z), \eta^{3}(7 z) \eta^{3}(z), \eta^{4}(4 z) \eta^{2}(2 z) \eta^{4}(z), \eta^{8}(2 z) \eta^{8}(z), \eta^{24}(z)$.

5.2. Метациклическая групша вида $\left\langle a, b: a^{7}=e, b^{6}=e, b^{-1} a b=a^{6}\right\rangle$. Эта группа порядка 63, она имеет 15 классов сопряженных элементов. У этой групшы 9 двумерных представлений, 6 одномерных представлений. Искомым представлением является прямая сумма, в которую входят все неприводимые представления с кратностью 1.

Элементам групшы соответствуют параболические формы: $\eta(21 z) \eta(3 z), \eta^{3}(7 z) \eta^{3}(z)$, $\eta^{4}(6 z), \eta^{8}(3 z), \eta^{12}(2 z), \eta^{24}(z)$.

5.3. Метациклическая групша вида $\left\langle a, b: a^{7}=e, b^{6}=e, b^{-1} a b=a^{3}\right\rangle$. Эта группа порядка 42, она имеет 7 классов сопряженных элементов, у нее имеется одно шестимерное представление и 6 одномерных.

Первым искомым представлением является прямая сумма всех неприводимых представлений, в которую 6-мерное неприводимое представление входит с кратностью 3 , одномерные представления - с кратностью 1.

Элементам групш соответствуют параболические формы: $\eta^{3}(7 z) \eta^{3}(z), \eta^{4}(6 z)$, $\eta^{8}(3 z), \eta^{12}(2 z), \eta^{24}(z)$.

Пусть

$$
T_{1}(a)=T_{1}(b)=1, \quad T_{2}(a)=1, \quad T_{2}(b)=-1,
$$

$T_{3}$ - 6-мерное неприводимое представление.

Второе допустимое представление $3\left(T_{1} \oplus T_{2} \oplus T_{3}\right)$.

Элементам группы соответствуют параболические формы: $\eta^{3}(7 z) \eta^{3}(z), \eta^{3}(6 z) \times$ $\eta^{3}(2 z), \eta^{6}(3 z) \eta^{6}(z), \eta^{8}(2 z) \eta^{8}(z), \eta^{24}(z)$.

5.4. Метациклическая групша вида $\left\langle a, b: a^{7}=e, b^{12}=e, b^{-1} a b=a^{3}\right\rangle$. Искомым представлением является представление

$$
T_{1} \oplus 2 T_{2} \oplus T_{3} \oplus 2 T_{4} \oplus 2 T_{5} \oplus T_{6},
$$

где

$$
\begin{gathered}
T_{k}(a)=1, \quad T_{k}(b)=i^{k}, k=1, \ldots, 4, \\
T_{5}(a)=\left(\begin{array}{cccccc}
\zeta_{7} & 0 & 0 & 0 & 0 & 0 \\
0 & \zeta_{7}{ }^{3} & 0 & 0 & 0 & 0 \\
0 & 0 & \zeta_{7}^{2} & 0 & 0 & 0 \\
0 & 0 & 0 & \zeta_{7}^{6} & 0 & 0 \\
0 & 0 & 0 & 0 & \zeta_{7}^{4} & 0 \\
0 & 0 & 0 & 0 & 0 & \zeta_{7}^{5}
\end{array}\right), \quad T_{7}(b)=\left(\begin{array}{cccccc}
0 & 0 & 0 & 0 & 0 & 1 \\
1 & 0 & 0 & 0 & 0 & 0 \\
0 & 1 & 0 & 0 & 0 & 0 \\
0 & 0 & 1 & 0 & 0 & 0 \\
0 & 0 & 0 & 1 & 0 & 0 \\
0 & 0 & 0 & 0 & 1 & 0
\end{array}\right), \\
T_{6}=T_{5} \otimes T_{1} .
\end{gathered}
$$

Элементам групшы соответствуют параболические формы: $\eta(14 z) \eta(7 z) \eta(2 z) \eta(z)$, $\eta(12 z) \eta(6 z) \eta(4 z) \eta(2 z), \quad \eta^{2}(6 z) \eta^{2}(3 z) \eta^{2}(2 z) \eta^{2}(z), \quad \eta^{4}(4 z) \eta^{2}(2 z) \eta^{4}(z), \quad \eta^{6}(3 z) \eta^{6}(z)$ $\eta^{3}(7 z) \eta^{3}(z), \eta^{8}(2 z) \eta^{8}(z), \eta^{24}(z)$. 
5.5. Метациклическая групша вида $\left\langle a, b: a^{7}=e, b^{3}=e, b^{-1} a b=a^{2}\right\rangle$. Искомьм представлением является прямая сумма представлений, в которую все неприводимые трехмерные представления входят с кратностью 3 , анеприводимые одномерные - с кратностью 2.

Элементам групшы соответствуют параболические формы: $\eta^{3}(7 z) \eta^{3}(z), \eta^{8}(3 z)$, $\eta^{24}(z)$.

Другим допустимым представлением является сумма трех 3-мерных представлений и шести тривиальных одномерных представлений.

Элементам группы соответствуют параболические формы: $\eta^{3}(7 z) \eta^{3}(z), \eta^{3}(6 z) \eta^{3}(2 z)$, $\eta^{24}(z)$.

5.6. Метациклическая групша вида $\left\langle a, b: a^{7}=e, b^{6}=e, b^{-1} a b=a^{2}\right\rangle$. Искомьм представлением является представление

$$
4 T_{1} \oplus 2 T_{2} \oplus T_{3} \oplus T_{4} \oplus 2 T_{5} \oplus 2 T_{6},
$$

где

$$
T_{1}(a)=1, \quad T_{1}(b)=1, \quad T_{2}(a)=1, \quad T_{2}(b)=-1,
$$

$T_{3}, T_{4}$ - 3-мерные представления, точные на элементе $b, T_{5}, T_{6}$ - 3 -мерные представления, не являющиеся точньги на элементе $b$.

Элементам групшы соответствуют параболические формы: $\eta(14 z) \eta(7 z) \eta(2 z) \eta(z)$, $\eta^{2}(6 z) \eta^{2}(3 z) \eta^{2}(2 z) \eta^{2}(z), \eta^{6}(3 z) \eta^{6}(z), \eta^{3}(7 z) \eta^{3}(z), \eta^{8}(2 z) \eta^{8}(z), \eta^{24}(z)$.

6. Метациклические группы вида $\left\langle a, b: a^{11}=e, b^{s}=e, b^{-1} a b=a^{r}\right\rangle$.

6.1. Метациклическая групша вида $\left\langle a, b: a^{11}=e, b^{20}=e, b^{-1} a b=a^{2}\right\rangle$. Эта группа имеет порядок 220, у нее 20 одномерных представлений и 2 неприводимых 10-мерных.

Искомым представлением является представление

$$
T_{1} \oplus T_{2} \oplus T_{3} \oplus T_{4} \oplus T_{5} \oplus 2 T_{6},
$$

где

$$
T_{k}(a)=1, \quad T_{k}(b)=i^{k}, \quad k=1, \ldots, 4,
$$

$T_{5}$ - 10-мерное представление, точное на элементе $b, T_{6}-10$-мерное представление, не являющееся точным на элементе $b$.

Элементам группы соответствуют параболические формы: $\eta(22 z) \eta(2 z), \eta^{2}(11 z) \eta^{2}(z)$, $\eta(20 z) \eta(4 z), \eta^{2}(10 z) \eta^{2}(z), \eta^{4}(5 z) \eta^{4}(z), \eta^{6}(4 z), \eta^{12}(2 z), \eta^{24}(z)$.

Эта группа содержит в качестве подгруппы следующие группы, которые, следовательно, также являются допустимьми: $\left\langle a, b: a^{11}=e, b^{10}=e, b^{-1} a b=a^{4}\right\rangle,\langle a, b$ : $\left.a^{11}=e, b^{5}=e, b^{-1} a b=a^{5}\right\rangle,\left\langle a, b: a^{11}=e, b^{4}=e, b^{-1} a b=a^{10}\right\rangle$.

6.2. Метациклическая групша вида $\left\langle a, b: a^{11}=e, b^{10}=e, b^{-1} a b=a^{2}\right\rangle$. Эта группа имеет порядок 110, у нее 10 одномерных представлений, одно неприводимое 10-мерное.

Искомым представлением является представление

$$
2 T_{1} \oplus 2 T_{2} \oplus 2 T_{3},
$$

где

$$
T_{k}(a)=1, \quad T_{k}(b)=(-1)^{k}, \quad k=1,2,
$$

$T_{3}-10$-мерное неприводимое представление.

Элементам группы соответствуют параболические формы: $\eta^{2}(11 z) \eta^{2}(z), \eta^{2}(10 z) \times$ $\eta^{2}(z), \eta^{4}(5 z) \eta^{4}(z), \eta^{12}(2 z), \eta^{24}(z)$. 
7. Метациклические группы вида $\left\langle a, b: a^{23}=e, b^{s}=e, b^{-1} a b=a^{r}\right\rangle$. В этом пункте мы рассмотрим только одну групу: все допустимые группы с таким генетическим кодом являются ее подгруппами.

7.1. Метациклическая групша вида $\left\langle a, b: a^{23}=e, b^{22}=e, b^{-1} a b=a^{5}\right\rangle$. Эта группа имеет порядок 506, у нее 23 одномерные представлений, одно неприводимое 22-мерное.

Искомым представлением является представление

$$
T_{1} \oplus T_{2} \oplus T_{3}
$$

где

$$
T_{k}(a)=1, \quad T_{k}(b)=(-1)^{k}, \quad k=1,2,
$$

$T_{3}-22$-мерное неприводимое представление.

Элементам грушшы соответствуют параболические формы: $\eta(23 z) \eta(z), \eta(22 z) \eta(2 z)$, $\eta^{2}(11 z) \eta^{2}(z), \eta^{12}(2 z), \eta^{24}(z)$.

Эта группа содержит в качестве подгруппы группы: $\left\langle a, b: a^{23}=e, b^{11}=e, b^{-1} a b=\right.$ $\left.a^{1} 0\right\rangle, D_{23}$.

\section{8. Метациклические группы, не имеющие представлений допустимого} типа. В этом пункте мы рассмотрим групш, элементы которых имеют допустимые порядки, но для самих групा не существует представлений искомого типа. Отсутствие искомого представления доказывается на основе анализа таблицы неприводимых представлений групы, причем не обязательно рассматривать представления для всех элементов групшы.

Все эти значения указаны в следующей таблице:

\begin{tabular}{|c|c|c|c|c|c|c|c|c|c|c|c|c|c|c|c|}
\hline$m$ & 3 & 3 & 3 & 3 & 4 & 4 & 4 & 4 & 5 & 5 & 5 & 7 & 7 & 7 & 11 \\
\hline$s$ & 10 & 14 & 16 & 24 & 12 & 14 & 16 & 20 & 6 & 10 & 20 & 14 & 18 & 9 & 22 \\
\hline$r$ & 2 & 2 & 2 & 2 & 3 & 3 & 3 & 3 & 2 & 2 & 2 & 6 & 3 & 2 & 10 \\
\hline
\end{tabular}

В качестве примера приведем доказательство для группы $\left\langle a, b: a^{3}=e, b^{16}=e\right.$, $\left.b^{-1} a b=a^{2}\right\rangle$.

Для этой группы не существует представления искомого типа, так как если у линейного оператора $T\left(a b^{2}\right)$ мы получим искомый набор собственных значений (это все корни степени 24 из 1), то среди собственных значений оператора $T(b)$, соответствуюших двумерным неприводимым представлениям, входящим в искомое представление, содержатся все первообразные корни степени 16 из 1, но в искомое представление необходимо также включить одномерные представления, которые переводят элемент $b$ в первообразные корни степени 16 из 1. Получим противоречие, так как элемент $b$ должен соответствовать форме $\eta(16 z) \eta(8 z)$.

Для других групп доказательство проводится аналогичными рассуждениями. 


\section{СПИСОК ЦИТИРОВАННОЙ ЛИТЕРАТУРЫ}

[1] Воскресенская Г. В.Модулярные формы и представления групп // Матем. заметки. 1992. T. 52. № 1. C. $25-31$.

[2] Воскресенская Г. В. Параболические формы и конечные подгруппы в $S L(5, \mathbb{C}) / /$ Функцион. анализ и его прилож. 1995. Т. 29. № 2. С. 71-73.

[3] Воскресенская Г. В. Модулярные формы и регулярные представления групп порядка 24 // Матем. заметки. 1996. Т. 60. № 2. С. 292-294.

[4] Воскресенская Г. В. Модулярные формы и представления групп диэдра // Матем. заметки. 1998. Т. 63 . № 1 . С. 130-133.

Самарский государственный университет 